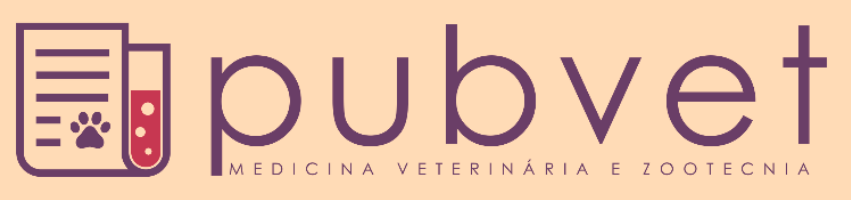

https://doi.org/10.31533/pubvet.v14n3a522.1-8

\title{
Diagnóstico e terapia de urolitíase em um felino: relato de caso
}

\author{
Patrícia Kelly Galúcio e Silva ${ }^{1^{*}}$, Edileni Barbosa da Silveira ${ }^{1}$, Dennis José da Silva Lima $^{2}{ }^{\bullet}$, Yuri \\ Teiichi da Silva Kobayashi ${ }^{30}$
}

${ }^{I}$ Discente de Medicina Veterinária da Universidade da Amazônia, Campus Santarém-PA, Brasil.

${ }^{2}$ Docente na Universidade da Amazônia, Campus Santarém-PA, Brasil.

${ }^{3}$ Médico Veterinário na Clínica Veterinária da Unama, Campus Santarém-PA, Brasil.

*Autor para correspondência, E-mail: patricia.kgs@hotmail.com

\begin{abstract}
Resumo. A urolitíase é uma afecção comum na clínica de felinos, caracterizada pela presença de urólitos em qualquer segmento do trato urinário. Este trabalho objetivou relatar o caso de um felino, macho, castrado, sem raça definida, 3 anos de idade, com ocorrência de dois urólitos na bexiga, apresentava hematúria, polaquiúria, disúria, estrangúria, incontinência urinária e apatia. No exame ultrassonográfico, foi detectado a presença de duas estruturas hiperecóicos na vesícula urinária. O tratamento foi à excisão cirúrgica, por intermédio da cistotomia. Logo após, os cálculos foram encaminhados para avaliação laboratorial qualitativa, na qual a composição designou cálculos de fosfato triplo/estruvita. No pós-operatório recomendou-se dieta a base de ração terapêutica específica para trato urinário. Após 6 meses o animal não manifestava sinais de doença do trato urinário. $\mathrm{O}$ procedimento cirúrgico mostrou eficiência eliminando a sintomatologia resultante dos urólitos. Além disso, a análise dos cálculos é essencial para estabelecer a dieta adequada e prevenir reicidivas.
\end{abstract}

Palavras chave: análise, cálculos vesicais, cistotomia, dieta, trato urinário

\section{Diagnostic and therapeutic approach of urolithiasis in a feline: case report}

\begin{abstract}
Urolithiasis is a common condition in the feline clinic, characterized by the presence of urolith in any segment of the urinary tract. This study aimed to report the case of a 3 - year - old male, castrated male with two bladder uroliths, hematuria, polaquiuria, dysuria, strangury, urinary incontinence and apathy. On the ultrasound examination, the presence of two hyperechoic structures was detected in the urinary vesicle. The treatment was surgical excision, througt cystotomy. Subsequently, the calculations were sent to a qualitative laboratory evaluation, in which the composition designated triple phosphate/ struvite stones. In the postoperative period, a diet based on specific therapeutic ration for the urinary tract was recommended. After 6 months the animal showed no signs of urinary tract disease. The surgical procedure showed efficiency eliminating the symptoms resulting from the uroliths. In addition, analysis of cauculations is essential to establish proper diet and prevent recidivism.
\end{abstract}

Keywords: analyze, bladder stones, cystotomy, diet, urinary tract

\section{Enfoque diagnóstico y terapéutica de urolitiasis en felino: relato de caso}

Resumen. La Urolitiasis es una afección común en la clínica de felinos, caracterizada por la presencia de urolitos en cualquier segmento del tracto urinario. Este trabajo tuvo como objetivo relatar el caso de un felino, macho, castrado, criollo, 3 años, con dos urolitos en la vejiga, presentaba hematuria, polaquiuria, disuria, estranguria, incontinencia urinaria y apatía. En el examen ultrasonográfico, se detectó la presencia de dos estructuras 
hiperecóicos en la vesícula urinaria. El tratamiento fue a la escisión quirúrgica, por intermedio de la cistotomía. Luego, los cálculos fueron encaminados para evaluación de laboratorio cualitativa, en la cual la composición designó cálculos de fosfato triple/ estruvita. En el postoperatorio se recomendó dieta a base de ración terapéutica específica para el tracto urinario. Después de 6 meses, el animal no muestro signos de enfermedad del tracto urinario. El procedimiento quirúrgico mostró eficiencia eliminando la sintomatología resultante de los urólitos. Además, el análisis de los cálculos es esencial para establecer la dieta adecuada y prevenir recidivas.

Palabras clave: análisis, cálculos vesicales, cistotomia, dieta, tracto urinario

\section{Introdução}

A urolitíase é a presença de cálculos no sistema urinário, sendo diagnosticada cada vez mais na rotina clínica veterinária (Grauer, 2015; Nelson \& Couto, 2015; Slatter, 2007). Quando a urina se torna supersaturada podem se formar urólitos e interferir na micção normal (Koehler et al., 2009) que são constituídos por agregados de solutos urinários precipitados e organizados em um núcleo central (ninho ou núcleo de cristal), e contêm principalmente cristalóides orgânicos e inorgânicos (Ettinger et al., 2017), que, por sua vez, são envolvidos por lâminas concêntricas e cristais de superfícies (Maxie \& Jubb, 2007; Zachary et al., 2012). Os cálculos em gatos ocorrem com maior frequência na bexiga e uretra (Gerber et al., 2005; Houston et al., 2016).

A formação dos urólitos está relacionada a fatores dietéticos e não dietéticos (Carciofi, 2008). O fator dietético está relacionado à composição da dieta, já que a mesma afeta a densidade específica, o volume e o pH urinário (Carciofi, 2008). Entre os não dietéticos estão a raça, idade, infecção do trato urinário, sexo (Elliott \& Lefebvre, 2009), obesidade, genética, sedentarismo, fatores regionais, demográficos e clima (Picavet et al., 2007).

Os sinais clínicos frequentemente encontrados em pacientes com urólitos vesicais são hematúria, iscúria, disúria e incontinência urinária e, em casos de complicações, podendo evoluir para obstrução uretral e/ou vesical, hidroureter e hidronefrose, ruptura vesical ou uretral, pielonefrite, uretrite e dilatação vesical (Inkelmann et al., 2011). Também podem estar presentes sinais sistêmicos como vômito, anorexia e depressão (Langston et al., 2008).

O diagnóstico de urolitíase é baseado no histórico do animal, exame físico, achados nos exames laboratoriais, radiográficos e/ou ultrassonográficos (Bartges \& Callens, 2015; Langston et al., 2008). Os exames complementares são necessários para auxiliar o diagnóstico podendo, e por desses, executar o diagnóstico diferencial entre urolitíase e outras enfermidades como infecções do trato urinário, coágulos, pólipos inflamatórios, inflamações ganulomatosas, neoplasias e anormalidades urogenitais $\underline{\text { (Osborne et }}$ al., 1986).

O tratamento dessa afecção pode ser clínico, através da dissolução e/ou interrupção do desenvolvimento subsequente de urólitos, ou cirúrgicos, através da remoção dos mesmos (Rick et al., 2017). No entanto, dependendo da diversidade de fatores, é necessário realizar a descompressão vesical e desobstrução uretral (Fossum, 2014). A profilaxia é baseada no conhecimento da composição mineral, a fim de se evitar recorrências (Fossum, 2014).

Objetivou-se relatar nesse trabalho a abordagem diagnóstica e terapêutica de urolitíase em um felino, na cidade de Santarém, Pará.

\section{Descrição do caso clínico}

Foi atendido na Clínica Escola Veterinária da Universidade da Amazônia - UNAMA, no dia 29 de setembro de 2018, na cidade de Santarém, Pará, um felino, macho, sem raça definida, pelagem tigrado, castrado, 3 anos de idade e pesando 4,500 kg. Durante o atendimento clínico a tutora relatou que o adquiriu ainda filhote, coabitava com vários animais da mesma e outras espécies, acesso restrito à rua, vermifugação atualizada e imunoprofilaxia atrasada. Tinha alimentação livre em maior quantidade a ração seca e enlatada (úmida), também fornecia comida caseira sendo composta por peixe, ingestão de água normalmente (normodipsia). 
Na queixa principal relatou que aproximadamente um mês o paciente começou a apresentar disúria, estrangúria, hematúria, polaquiúria, periúria, incontinência urinária e apatia. Tentou tratamento com Enrofloxacino (Baytril $\left.{ }^{\circledR}\right)$ 15mg (1/2 VO/BID/ 7 dias), porém sem resultados. O animal possuía histórico anterior a doenças do trato urinário inferior dos felinos (DTUIF), já havia sido desobstruído 4 vezes. Ao exame físico apresentava a musculatura abdominal contraída, bexiga pouco repleta e tenesmo vesical. Apresentou frequência respiratória de 48 movimentos por minuto (mpm), frequência cardíaca de 94 batimentos por minuto (bpm), mucosas normocoradas, tempo de preenchimento capilar de 2 (segundos), desidratação discreta de até $5 \%$, temperatura corporal de $37.2^{\circ} \mathrm{C}$, linfonodos palpáveis e não reativos. Foram solicitados exames complementares como hemograma, perfil bioquímico sérico, urinálise (amostra colhida por cistocentese) e ultrassonografia abdominal. No hemograma completo, o eritrograma não revelou alterações (Tabela 1), entretanto o leucograma evidenciou monocitose e o plaquetograma apresentou plaquetopenia (Tabela 2). Na avaliação bioquímica sérica foi observado azotemia (Tabela 3).

Tabela 1. Hemograma - Eritrograma de um felino, macho, 3 anos, realizado pelo laboratório Vetanálises.

\begin{tabular}{lcc}
\hline Parâmetros & Resultados & Valores de Referência \\
\hline Hemácias & $8.5910^{3} / \mathrm{mm}^{3}$ & 5,0 a 10,0 \\
Hemoglobina & $12,4 \mathrm{~g} / \mathrm{dL}$ & 8,0 a 15,0 \\
Hematócrito & $37,1 \%$ & 24 a 45 \\
V.C.M. & $43 \%$ & 39 a 55 \\
H.C.M. & $14 \mathrm{pg}$ & 12,5 a 17,5 \\
C.H.C.M. & $33 \%$ & 30 a 36 \\
Metarrubrícitos & 0 und & 0 \\
\hline
\end{tabular}

Tabela 2. Hemograma - Leucograma de um felino, macho, 3 anos, realizado pelo laboratório Vetanálises.

\begin{tabular}{|c|c|c|c|c|}
\hline Parâmetros & Relativo (\%) & Absoluto & Relativo & Absoluto \\
\hline Leucócitos Totais & & $19.500 / \mathrm{mm}^{3}$ & & 5.500 a 19.500 \\
\hline Miélocitos & $0 \%$ & $0 / \mathrm{mm}^{3}$ & 0 a 0 & 0 a 0 \\
\hline Metamielócitos & $0 \%$ & $0 / \mathrm{mm}^{3}$ & 0 a 0 & 0 a 0 \\
\hline Bastonetes & $0 \%$ & $0 / \mathrm{mm}^{3}$ & 0 a 3 & 0 a 500 \\
\hline Neutrófilos & $72 \%$ & $14.040 / \mathrm{mm}^{3}$ & 32 a 75 & 1.925 a 14.625 \\
\hline Basófilos & $0 \%$ & $0 / \mathrm{mm}^{3}$ & 0 a 1 & 0 a 195 \\
\hline Eosinófilos & $5 \%$ & $975 / \mathrm{mm}^{3}$ & 2 a 12 & 110 a 2.340 \\
\hline Linfócitos & $18 \%$ & $3.510 / \mathrm{mm}^{3}$ & 30 a 48 & 1.800 a 7.680 \\
\hline Monócitos & $5 \%$ & $975 / \mathrm{mm}^{3}$ & 1 a 4 & 55 a 780 \\
\hline Plaquetas & & $206.000 / \mathrm{mm}^{3}$ & & 250.000 a 800.000 \\
\hline
\end{tabular}

(Macroplaquetas +)

Tabela 3. Perfil Bioquímico - Função Renal de um felino, macho, 3 anos, realizado pelo laboratório Vetanálises.

\begin{tabular}{lcc}
\hline Parâmetros & Resultados & Valores de Referência \\
\hline Creatinina & $1,98 \mathrm{mg} / \mathrm{dL}$ & $0,70 \mathrm{a} 1,80 \mathrm{mg} / \mathrm{dL}$ \\
Uréia & $92 \mathrm{mg} / \mathrm{dL}$ & $10 \mathrm{a} 60 \mathrm{mg} / \mathrm{dL}$ \\
\hline
\end{tabular}

A urinálise apresentava alterações, no exame físico a coloração apresentava-se de forma avermelhada e aspecto turvo, no exame químico o pH encontrava-se dentro dos parâmetros normais, positivo para a presença de nitrito e proteínas. No exame de sedimentos as hemácias apresentavam-se de forma incontáveis, leucocitose, frequência de células escamosas e bactérias (Tabela 4). Como exame de imagem a ultrassonografia abdominal foi eleita para o detalhamento e confirmação do diagnóstico. Nenhuma anormalidade foi encontrada, exceto a vesícula urinária que se apresentava parcialmente repleta com conteúdo anecóico, paredes espessadas, apresentando duas estruturas hiperecóicas e formadoras de sombra acústica em seu interior, o maior com 0,8 e menor com 0,5 milímetros de diâmetro em topografia de bexiga, sugestivo de urolitíase vesical (Figura 1). Estando os outros parâmetros dentro da normalidade para a espécie. 
Tabela 4. Urinálise de um felino, macho, 3 anos, realizado pelo laboratório Vetanálises.

\begin{tabular}{|c|c|c|}
\hline Exame Físico & Resultados & Valores de Referência \\
\hline$\overline{\text { Volume }}$ & 3,5 & $10,0 \mathrm{~mL}$ \\
\hline Cor & Avermelhado & Amarelo citrino \\
\hline Aspecto & Turvo & Límpido \\
\hline Odor & Sui Generis & Sui Generis \\
\hline Densidade & 1.030 & 1.015 a 1.040 \\
\hline \multicolumn{3}{|l|}{ Exame Químico } \\
\hline$\overline{\mathrm{pH}}$ & 7,0 & 5,5 a 7,5 \\
\hline Nitrito & Positivo & Negativo \\
\hline Proteínas & Positivo & Negativo a Traços \\
\hline Glicose & Negativo & Negativo \\
\hline Cetonas & Negativo & Negativo \\
\hline Bilirrubinas & Negativo & Negativo \\
\hline Urobilinogênio & Normal $\mu \mathrm{mol} / \mathrm{L}$ & $\operatorname{Normal}(3,2 \mu \mathrm{mol} / \mathrm{L})$ \\
\hline Sangue & Negativo & Negativo \\
\hline \multicolumn{3}{|l|}{ Exame do Sedimento } \\
\hline Hemácias & Incontáveis & 01 a $10 /$ campo 400x \\
\hline Leucócitos & 32 & 01 a $10 /$ campo $400 x$ \\
\hline Células Escamosas & Frequentes & Ausentes a Raras \\
\hline Células Caudadas & Ausentes & Ausentes \\
\hline Células Tubulares & Raras & Ausentes \\
\hline Cilindros Hialinos & Ausentes & Ausentes a Raras \\
\hline Cilindros Celulares & Ausentes & Ausentes \\
\hline Cilindros Granulares & Ausentes & Ausentes \\
\hline Cilindros lipídicos & Ausentes & Ausentes \\
\hline Cilindros Cerosos & Ausentes & Ausentes \\
\hline Muco & Ausentes & Ausentes \\
\hline Bactéria & Frequentes & Ausentes a Raras \\
\hline Cristais & Ausentes & Ausentes a Raras \\
\hline
\end{tabular}

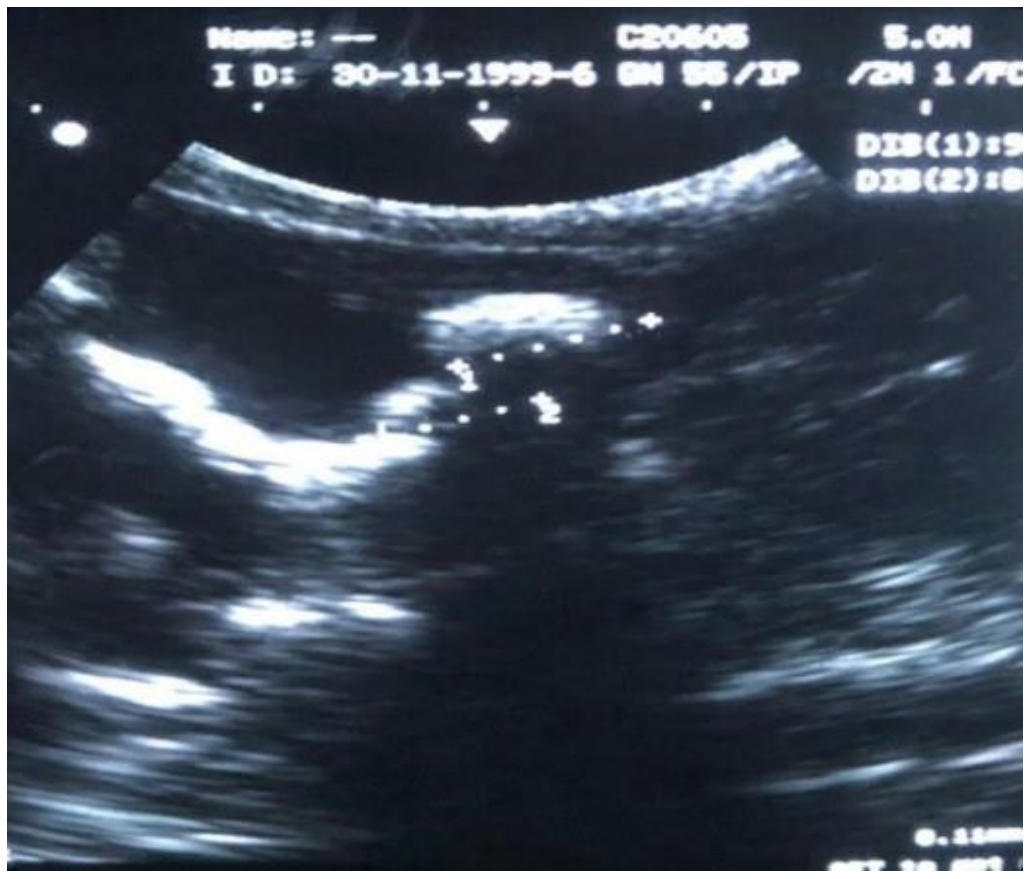

Figura1. Imagem ultrassonográfica de duas estruturas hiperecóicas formadoras de sombra acústica na vesícula urinaria. 
Para terapia sintomática foi realizada a antibioticoterapia com amoxicilina associada ao clavulanato de potássio $\left(\right.$ Clavulin $\left.^{\circledR}\right)(62,5 \mathrm{mg} /$ gato $\mathrm{VO} / \mathrm{BID} / 14 \mathrm{~d})$, cetoprofeno (Cetofenid $\left.{ }^{\circledR}\right)(1 \mathrm{mg} / \mathrm{kg} \mathrm{VO} / \mathrm{SID} / 3 \mathrm{~d})$. Após a realização dos exames, o paciente foi encaminhado para o procedimento cirúrgico. Foi submetido a cistotomia para remoção dos cálculos vesicais no dia 09 de outubro de 2018. Após o jejum hídrico e alimentar, foi realizado o preparo do paciente, com tricotomia na região abdominal, anestesia, fluidoterapia, posicionamento em decúbito dorsal e antissepsia da pele.

$\mathrm{Na}$ medicação pré-anestésica foi utilizado acepromazina $(0,1 \mathrm{mg} / \mathrm{kg})$ associado ao tramadol $(4 \mathrm{mg} / \mathrm{kg} / \mathrm{IM}) \operatorname{logo}$ em seguida, dipirona $(20 \mathrm{mg} / \mathrm{kg} / \mathrm{SC})$. Como indução anestésica foi utilizado quetamina $(5 \mathrm{mg} / \mathrm{kg})$ associado ao diazepam $(0,5 \mathrm{mg} / \mathrm{kg} \mathrm{IV})$ e fluidoterapia com cloreto de sódio a $0,9 \%$ $(150 \mathrm{~mL} / \mathrm{IV})$. Posteriormente incisou-se a pele, isolando a bexiga da cavidade abdominal com compressas cirúrgicas, coletando o pouco de urina que havia no lúmen da vesícula através de cistocentese, aplicaram-se duas suturas de retenção nas extremidades lateral direta e lateral esquerda para facilitar abertura da bexiga e permitir a inspeção interior (Figura 2). Fez-se então uma incisão estocada na superfície ventral da mesma.

Após a omentalização à bexiga, o abdome foi fechado como de rotina, com fio nylon 3-0 e pontos isolados simples. Para o pós-operatório, foi prescrito antibiótico a base de amoxicilina associado ao clavulanato de potássio $\left(\right.$ Clavulin $\left.^{\circledR}\right)\left(62,5 \mathrm{mg} /\right.$ gato VO/BID/14d), cetoprofeno (cetofenid $\left.{ }^{\circledR}\right)(1 \mathrm{mg} / \mathrm{kg}$ $\mathrm{VO} / \mathrm{SID} / 3 \mathrm{~d})$, cloridrato de amitriptilina ( $25 \mathrm{mg} / \mathrm{kg} \mathrm{VO} / \mathrm{SID} / 60 \mathrm{~d})$, limpeza com solução fisiológica e logo em seguida, aplicar rifocina sobre a ferida cirúrgica com auxílio de gaze (BID/5d), pomada cicatrizante tópica (BID/5d) e ração terapêutica para trato urinário felino (Royal Canin-Urinary $S / \mathrm{O}^{\circledR}$ ) até novas recomendações. Os cálculos (Figura 3 ) foram encaminhados para análise laboratorial, apresentaram as composições de carbonato, magnésio e amônio (Tabela 5).

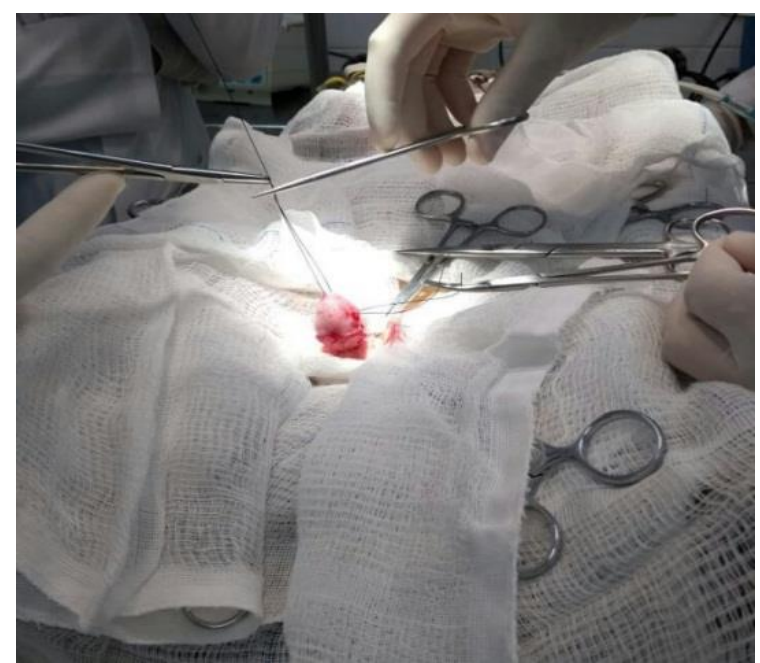

Figura 2. Aplicação de duas suturas de retenção nas extremidades na bexiga

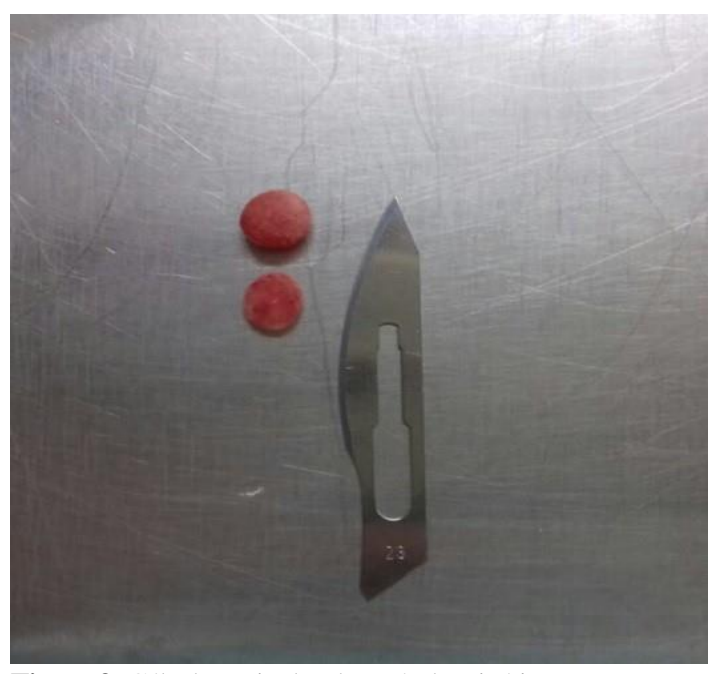

Figura 3. Cálculos retirados da vesícula urinária.

Tabela 5. Análise qualitativa dos cálculos urinários de um felino, macho, 3 anos, realizado pelo laboratório Vetanálises.

\begin{tabular}{|c|c|c|c|}
\hline \multirow{2}{*}{$\begin{array}{l}\text { Análise Física } \\
\text { Pesos: } 0,21 \mathrm{~g} \mathrm{e} 0,1 \mathrm{~g}\end{array}$} & \multicolumn{3}{|c|}{ Análise Química } \\
\hline & Carbonato: & + & Urato: - \\
\hline Dimensões: $5 \mathrm{x} 4 \mathrm{~mm}$ e $7 \times 5 \mathrm{~mm}$ & Oxalato: & - & Cistina: - \\
\hline Formas: arredondadas & Fosfato: & - & \\
\hline Cores: marrons & Cálcio: & - & \\
\hline Superfícies: lisas & Magnésio: & +++ & \\
\hline Consistências: macias & Amônio: & +++ & \\
\hline
\end{tabular}

(Análise qualitativa dos componentes representada em cruzes: + à ++++).

Retornou no dia 16 de outubro de 2018 para retirada dos pontos, o paciente apresentava-se com mucosas normocoradas, normohidratado, TC: $37.8^{\circ}$. Após 6 meses o animal retornou para reavaliação, 
e não manifestava sinais clínicos de urolitíase. No entanto, não foram realizados exames complementares devido as condições financeiras do tutor.

\section{Discussão}

O presente relato descreve um caso de urolitíase (presença de urólito na bexiga que é denominado cistolitíase). O índice maior de urolitíase ocorre entre dois e sete anos de idade (Cannon et al., 2007; Grauer, $\underline{2015}$ ). Os urólitos dessa composição são tipicamente encontrados em felinos das raças Himalaios, Persa e o gato doméstico comum (Cannon et al., 2007; Gerber et al., 2005). Segundo Grauer (2015) a castração tanto de machos quanto de fêmeas contribui para obesidade e a diminuição da atividade física, tornando-os mais sedentários. Desse modo, os achados obtidos neste relato estão de acordo com estudos publicados.

O paciente em estudo possuía alimentação à vontade. De acordo com Palma et al. (2009) a dieta é um fator que pode favorecer a formação de cálculos quando associada a um consumo excessivo de ração, resultando em obesidade e maior quantidade de excreção de minerais na urina. Em nosso estudo, o paciente também tinha acesso restrito à rua, isto corrobora com a literatura, haja vista que, os animais que não possuem acesso à rua urinam menos e retém mais urina, considerando que a hipoatividade promove a diminuição da frequência de micção, alcalinizando o $\mathrm{pH}$, favorecendo a formação de cristais e, posteriormente, de cálculos (Ettinger et al., 2017; Feldman, 1997; Gunn-Moore, 2003; Hostutler et al., 2005).

Os sinais clínicos apresentados foram hematúria, polaquiúria, disúria, estrangúria, incontinência urinária e micção em local inapropriado, sinais que também foram observados por (Langston et al., 2008). Além das predisposições mencionadas, o paciente possuía fatores de risco para formação e desenvolvimento de urólitos, pois o mesmo já tinha histórico anterior a DTUIF. Independente da terapia e recuperação, observou-se em estudos que cerca de 35 a $50 \%$ dos gatos, apresentam uma ou mais reicidivas principalmente nos primeiros meses após o episódio inicial (Reche Junior \& Hagiwara, 2004).

O diagnóstico realizado teve como base a história clínica, exame físico, e exames complementares, assim como descrito por Grauer (2015). Em pacientes diagnosticados com urolitíase, espera-se encontrar na urinálise, grande quantidade de hemácias, além de leucócitos e bactérias produtoras ou não de urease (Houston et al., 2004). Contudo, apesar do tenesmo vesical, o paciente conseguia eliminar pequena quantidade de urina, mas apresentava azotemia que poderia ser justificada pela obstrução parcial do fluxo urinário, ocasionando acúmulo desses metabólitos na corrente sanguínea. O paciente permaneceu na fluidoterapia, porque a fluidoterapia é o componente mais importante da terapia para animais com azotemia pós-renal, restabelecer o fluxo urinário junto a fluidoterapia apropriada pode resolver a hipercalemia, azotemia e acidose metabólica para a maioria dos pacientes (Hostutler et al., 2005). Ademais, foi observado monocitose e proteína total aumentada associada a função renal diminuída, indicando a existência de um provável processo inflamatório crônico no trato urinário.

Vários tipos de urólitos podem se formar no trato urinário inferior do felino, tais como o urato de amônio, o fosfato de cálcio, a cistina, a sílica, mistos (Kaufmann et al., 2011; Lazzarotto, 2000), ou sangue seco solidificado (Westropp et al., 2006). Nesse caso relatado, o urólito analisado confirmou ser do tipo estruvita, tal informação obtida pela análise qualitativa. Foi possível visualizar amônio e magnésio em maiores quantidades, mas também foi observado fosfato em menor quantidade. Segundo Fossum (2014), os urólitos de estruvita são compostos por fosfato, amônio e magnésio, sendo a cristalúria por estruvita a mais comum nos felinos (Buffington et al., 1997).

Baseado nas evidências diagnosticado na história clínica e achado aos exames optou-se pela cirurgia de cistotomia como prioridade imediata assim como preconizado por Galvão et al. (2010) que a intervenção cirúrgica é recomendada para o felino que apresenta episódios recorrentes a obstrução. Com o diagnóstico e tratamento estipulado, o animal possuiu um prognóstico de bom a reservado. $\mathrm{O}$ tratamento clínico tem por finalidade dissolver e/interromper o crescimento dos urólitos pequenos através da diminuição de sais calculogênicos na urina associado à antibioticoterapia (realizada após urocultura e antibiograma) (Grauer, 2015; Monferdini \& Oliveira, 2009). No caso citado, por não ter realizado urocultura e antibiograma por falta de recursos da tutora, optou-se pelo uso da amoxicilina com clavulanato de potássio, sendo estes indicados para infecções do trato urinário (Papich, 2012). 
Alguns estudos demonstram que os felinos são mais sensíveis ao estresse, devido a mudanças em seu ambiente (Silva et al., 2013). De acordo com essa afirmação, recomendou-se o uso da amitriptilina, um fármaco antidepressivo tricíclico e ansiolítico (com propriedades anticolinérgicas, anti-histamínicas, anti- $\alpha$-adrenérgicas, antiinflamatórias e analgésicas) - defendida empiricamente para tratar gatos com sinais persistentes (Tilley et al., 2008). Uma vez que o paciente relatado vivia em ambiente superpopuloso, e o manejo nutricional para o paciente objetivou melhorar a qualidade alimentar trocando a dieta por uma ração urinária específica de alto valor biológico. Papich (2012) afirma que a mudança na dieta de pacientes com cálculos de estruvita é essencial para reduzir a concentração de uréia, fósforo e magnésio na urina. De acordo com Palma et al. (2009) a dieta é um fator que pode favorecer a formação de cálculos quando associada a um consumo excessivo de ração, resultando em obesidade e maior quantidade de excreção de minerais na urina.

\section{Conclusão}

A urolitíase não deve ser considerada uma enfermidade única, com causa pontual, e sim sequela de múltiplas desordens sistêmicas em interação. Nesse caso, pelo histórico, diagnóstico, procedimento cirúrgico e tratamento pós-operatório, mostraram-se eficientes, obtendo a recuperação do animal. Portanto, identificar os mecanismos de litíase urinária auxilia no diagnóstico precoce e na elaboração da terapia adequada para reduzir de formação de urólitos, evitar sua ocorrência e ainda prever as possíveis falhas no tratamento.

\section{Referências bibliográficas}

Bartges, J. W. \& Callens, A. J. (2015). Urolithiasis. Veterinary Clinics: Small Animal Practice, 45(4), 747-768.

Buffington, C. A. T., Chew, D. J., Kendall, M. S., Scrivani, P. V, Thompson, S. B., Blaisdell, J. L., \& Woodworth, B. E. (1997). Clinical evaluation of cats with nonobstructive urinary tract diseases. Journal of the American Veterinary Medical Association, 210(1), 46-50.

Cannon, A. B., Westropp, J. L., Ruby, A. L., \& Kass, P. H. (2007). Evaluation of trends in urolith composition in cats: 5,230 cases (1985-2004). Journal of the American Veterinary Medical Association, 231(4), 570-576.

Carciofi, A. C. (2008). Manejo nutricional do cão e do gato hospitalizado. In UNESP (Ed.), Apontamentos teóricos das disciplinas de Clínica das Doenças Carenciais, Endócrinas e Metabólicas e de Nutrição e Alimentação de Cães e Gatos. Universsidade de São Paulo.

Elliott, D. A., \& Lefebvre, H. (2009). Textbook of veterinary internal medicine. In P. Pibot, V. Biourge, \& D. A. Elliott (Eds.), Encyclopedia of canine clinical nutrition royal canin (pp. 252-282). Royal Canin.

Ettinger, S. J., Feldman, E. C. \& Cote, E. (2017). Textbook of Veterinary Internal Medicine-eBook. Elsevier Health Sciences.

Feldman, E. C. (1997). Tratado de medicina interna veterinária. In Moléstias do cão e do gato (Vol. 3).

Fossum, T. W. (2014). Cirurgia de pequenos animais (4th ed., Vol. 1). Elsevier Brasil.

Galvão, A. L. B., Ondani, A. C., Frazílio, F. O., \& Ferreira, G. S. (2010). Obstrução uretral em gatos machos-revisão literária. Acta Veterinaria Brasilica, 4(1), 1-6.

Gerber, B., Boretti, F. S., Kley, S., Laluha, P., Müller, C., Sieber, N., Unterer, S., Wenger, M., Flückiger, M., \& Glaus, T. (2005). Evaluation of clinical signs and causes of lower urinary tract disease in European cats. Journal of Small Animal Practice, 46(12), 571-577.

Grauer, G. (2015). Manifestações clínicas dos distúrbios urinários; Urolítiase canina. In A. W. Nelson \& C. G. Couto (Eds.), Medicina Interna de Pequenos Animais (Issue 1). Elsevier Editora.

Grauer, G. F. (2015). Feline Struvite \& Calcium Oxalate Urolithiasis. Todays Vet Pract, 5(5), 14-20.

Gunn-Moore, D. A. (2003). Feline lower urinary tract disease. Journal of Feline Medicine and Surgery, 5(2), 133-138.

Hostutler, R. A., Chew, D. J., \& DiBartola, S. P. (2005). Recent concepts in feline lower urinary tract disease. Veterinary Clinics: Small Animal Practice, 35(1), 147-170. 
Houston, D. M., Moore, A. E. P., Favrin, M. G., \& Hoff, B. (2004). Canine urolithiasis: a look at over 16000 urolith submissions to the Canadian Veterinary Urolith Centre from February 1998 to April 2003. The Canadian Veterinary Journal, 45(3), 225-230.

Houston, D. M., Vanstone, N. P., Moore, A. E. P., Weese, H. E., \& Weese, J. S. (2016). Evaluation of 21426 feline bladder urolith submissions to the Canadian Veterinary Urolith Centre (1998-2014). The Canadian Veterinary Journal, 57(2), 196-201.

Inkelmann, M. A., Kommers, G. D., Fighera, R. A., Irigoyen, L. F., Barros, C. S. L., Silveira, I. P., \& Trost, M. E. (2011). Neoplasmas do sistema urinário em 113 cães. Pesquisa Veterinária Brasileira, 31(11), 1102-1107.

Kaufmann, C., Neves, R. C., \& Habermann, J. C. A. (2011). Doença do trato urinário inferior dos felinos. Anuário Da Produção Científica Dos Cursos de Pós-Graduação, 4(4), 193-214.

Koehler, L. A., Osborne, C. A., Buettner, M. T., Lulich, J. P., \& Behnke, R. (2009). Canine uroliths: frequently asked questions and their answers. Veterinary Clinics of North America: Small Animal Practice, 39(1), 161-181.

Langston, C., Gisselman, K., Palma, D., \& McCue, J. (2008). Diagnosis of urolithiasis. Compendium, $30(8), 447-450$.

Lazzarotto, J. J. (2000). Doença do trato urinário inferior dos felinos associada aos cristais de estruvita. Revista Da FZVA, 7(1), 58-64.

Maxie, M. G., \& Jubb, K. (2007). Palmer's pathology of domestic animals. Philadelphia, 5(2), 523653.

Monferdini, R. P., \& Oliveira, J. (2009). Manejo nutricional para cães e gatos com urolitíase-Revisão bibliográfica. Acta Veterinaria Brasilica, 3(1), 1-4.

Nelson, R. W., \& Couto, C. G. (2015). Medicina interna de pequenos animais (Issue 1). Elsevier Editora.

Osborne, C. A., Clinton, C. W., Bamman, L. K., Moran, H. C., Coston, B. R., \& Frost, A. P. (1986). Prevalence of canine uroliths: Minnesota urolith center. Veterinary Clinics of North America: Small Animal Practice, 16(1), 27-44.

Palma, D., Langston, C., Gisselman, K., \& McCue, J. (2009). Feline struvite urolithiasis. Compendium Continuig Education for Veterinarians, 31(12), E1-7.

Papich, M. G. (2012). Manual Saunders de Terapia Veterinária. Elsevier Health Sciences Brazil.

Picavet, P., Detilleux, J., Verschuren, S., Sparkes, A., Lulich, J., Osborne, C. A., Istasse, L., \& Diez, M. (2007). Analysis of 4495 canine and feline uroliths in the Benelux. A retrospective study: 19942004. Journal of Animal Physiology and Animal Nutrition, 91(5-6), 247-251.

Reche Junior, A., \& Hagiwara, M. K. (2004). Semelhanças entre a doença idiopática do trato urinário inferior dos felinos e a cistite intersticial humana. Ciência Rural, 34(1), 315-321.

Rick, G. W., Conrad, M. L. H., Vargas, R. M., Machado, R. Z., Lang, P. C., Serafini, G. M. C., \& Bones, V. C. (2017). Urolitíase em cães e gatos. PUBVET, 11, 646-743.

Silva, A. C., Muzzi, R. A. L., Oberlender, G., Muzzi, L. A. L., Coelho, M. R., \& Henrique, B. F. (2013). Cistite Idiopática Felina: Revisão de Literatura. Arquivos de Ciências Veterinárias e Zoologia Da UNIPAR, 16(1), 93-96.

Slatter, D. H. (2007). Manual de cirurgia de pequenos animais. Manole.

Tilley, L. P., Smith, J. R., \& Francis, W. K. (2008). Consulta veterinária em 5 minutos: Espécies canina e felina. Editora Manole.

Westropp, J. L., Ruby, A. L., Bailiff, N. L., Kyles, A. E., \& Ling, G. V. (2006). Dried solidified blood calculi in the urinary tract of cats. Journal of Veterinary Internal Medicine, 20(4), 828-834.

Zachary, J. F., McGavin, D., \& McGavin, M. D. (2012). Bases da patologia em veterinária. Elsevier Brasil.

Recebido: 4 de setembro, 2019.

Aprovado: 8 de novembro, 2019.

Publicado: 21 de abril, 2020.
Licenciamento: Este artigo é publicado na modalidade Acesso Aberto sob a licença Creative Commons Atribuição 4.0 (CC-BY 4.0), a qual permite uso irrestrito, distribuição, reprodução em qualquer meio, desde que o autor e a fonte sejam devidamente creditados. 